\title{
Preoperative Assessment of Pulmonary Function Tests and Outcomes After Cardiac Surgery
}

\author{
Masahiro Fujii, MD, PhD, Dai Nishina, MD, Ryuzo Bessho, MD, PhD \\ Department of Cardiovascular Surgery, Nippon Medical School Chiba Hokusoh Hospital, Kamagari, Inzai, Chiba, Japan
}

\section{ABSTRACT}

Background: To investigate the association between preoperative pulmonary function evaluations and surgical outcomes of patients with chronic lung disease following cardiac surgery.

Methods: This retrospective observational study evaluated 148 patients using preoperative pulmonary function tests before undergoing cardiac surgery. Patients were divided into 4 groups (normal, obstructive, restrictive, and combined disorder), based on the result of the pulmonary function tests. Additionally, we evaluated the percent predicted forced expiratory volume in 1 second. Finally, we investigated the mechanical ventilation duration, length of postoperative hospital stay, and the 30-day mortality rate between the groups in each study.

Results: The mechanical ventilation duration and length of postoperative hospital stay in the combined group was significantly longer than that in the other groups $(P<.0001$, $P<.0001$, respectively). Patients in the restrictive group had a significantly longer postoperative ventilation or hospitalization than those in the normal group $(P=.0479, P=.0164$, respectively). However, there were no significant differences in the 30-day mortality rates between the groups. There also was a significant negative correlation between the percent predicted forced expiratory volume in 1 second and mechanical ventilation $\left(\mathrm{R}^{2}=0.052, P=.0054\right)$ and postoperative hospitalization $\left(\mathrm{R}^{2}=0.042, P=.0122\right)$.

Conclusion: Risk stratification by preoperative pulmonary function tests may be used to accurately identify the postoperative outcomes in chronic lung disease patients following cardiac surgery.

\section{INTRODUCTION}

In recent years, the age of and complications associated with cardiac patients have increased. The Japan System for Cardiac Operative Risk Evaluation (JapanSCORE), The Society of Thoracic Surgeons (STS) risk model, and European System for Cardiac Risk Evaluation (EuroSCORE) II widely have been used to assess the preoperative risk of cardiac surgery [Miyata

Received October 29, 2019; accepted April 9, 2020.

Correspondence: Masabiro Fujii, MD, PbD, Cardiovascular Surgery, Nippon Medical School Chiba Hokusob Hospital, 1715 Kamagari, Inzai, Chiba, 2701694, Japan; +81476991111; fax: +81476991921 (e-mail: m-fujii@nms.ac.jp).
2015; Shroyer 2003; Roques 1999]. Additionally, studies have reported that preoperative pulmonary dysfunction affects surgical outcomes. According to clinical history or spirometric criteria, chronic obstructive pulmonary disease (COPD) is present in $4 \%-26 \%$ of patients undergoing cardiac surgery [Roques 1999; Saleh 2012]; therefore, studies have suggested that COPD leads to an increased risk of respiratory and infectious complications and mortality following cardiac surgery [Samuels 1998]. Respiratory failure also has been identified as a common complication that occurs in $2 \%-10 \%$ in patients following cardiac surgery [Filsoufi 2008; Saleh 2012]. Additionally, previous studies have demonstrated that respiratory failure was associated with an increased length of hospital stay and postoperative morbidity and mortality, and this ultimately led to greater medical costs [Filsoufi 2008; Cohen 2000].

The following pulmonary function tests (PFTs) were measured: vital capacity (VC), forced vital capacity (FVC), vital capacity as a percent of predicted (\%VC), forced expiratory volume in the first second (FEV1.0), ratio between FEV1.0 and FVC (FEV1.0\%), and percent predicted FEV1.0 (\%FEV1.0). Although recent guidelines for the diagnosis of lung disease have utilized PFTs to diagnose and classify the severity of pulmonary function [Celli 2004], preoperative spirometry assessments have revealed differential diagnoses. Additionally, although some studies have denied the association between the stratification of COPD by spirometry and surgical outcomes because of overestimation [Magee 2013], Ad et al revealed that there was a significant underestimation of the incidence of adverse outcomes when spirometry was the only risk model used [Ad 2010].

Restrictive lung disease also is a significant predictor of earlier death in long-term follow-up [Mannino 2003]. However, few reports have examined the relationship between restrictive lung disease and postoperative outcomes following cardiac surgery. If FVC is reduced, FEV1.0\% may be overestimated, therefore, we need to focus on the \% FEV1.0 as a parameter.

In the current study, patients were divided into groups based on the severity of ventilation disorders using preoperative PFTs in order to determine the relationship between preoperative pulmonary function evaluations and surgical outcomes after cardiac surgery with cardiopulmonary bypass (CPB).

\section{MATERIALS AND METHODS}

Study population and design: One hundred forty-eight patients were enrolled in this retrospective observational 
Baseline characteristics and operative parameters

\begin{tabular}{|c|c|c|c|c|c|}
\hline Cases & 92 & 33 & 20 & 3 & - \\
\hline FEV1.0\%† & $78.5 \pm 5.3$ & $63.5 \pm 6.0$ & $78.4 \pm 6.5$ & $63.8 \pm 7.4$ & $<.0001$ \\
\hline Age (years) & $66 \pm 12$ & $71 \pm 7$ & $72 \pm 7$ & $76 \pm 4^{*}$ & .0108 \\
\hline Body surface area (m2) & $1.62 \pm 0.18$ & $1.62 \pm 0.18$ & $1.52 \pm 0.17$ & $1.34 \pm 0.10^{*}$ & .0095 \\
\hline Smoker (\%) & 45.7 & 69.7 & 15.0 & $0 *$ & .0005 \\
\hline Hypertension (\%) & 55.4 & 69.7 & 65.0 & 66.7 & .5016 \\
\hline Dyslipidemia (\%) & 53.3 & 60.6 & $20.0 *$ & 33.3 & .0243 \\
\hline Diabetes mellitus (\%) & 20.7 & 30.3 & 25.0 & 66.7 & .2365 \\
\hline Left ventricular ejection fraction (\%) & $63 \pm 10$ & $59 \pm 12$ & $58 \pm 14$ & $50 \pm 20 *$ & .0352 \\
\hline Operative time (minutes) & $335 \pm 125$ & $322 \pm 119$ & $328 \pm 102$ & $338 \pm 38$ & .9553 \\
\hline Cardiopulmonary bypass time (minutes) & $176 \pm 75$ & $170 \pm 68$ & $163 \pm 54$ & $188 \pm 25$ & .8512 \\
\hline Aortic clamping time (minutes) & $141 \pm 57$ & $135 \pm 55$ & $134 \pm 48$ & $140 \pm 27$ & .9274 \\
\hline
\end{tabular}

Data are presented as the mean \pm standard deviation or percentage.

$* P<0.05$ versus other groups

†Ratio between forced expiratory volume in the first second and forced vital capacity

study from April 2011 to March 2016. The following study protocol was approved by the Ethics Committee of our hospital (No. 729), and this study was registered with the University Hospital Medical Information Network (study ID: UMIN000037512). Owing to the retrospective nature of the study and due to the fact that data collection was a routine patient practice in our hospital, the requirement for informed consent was waived, but patients also were given the option to opt out of the study. Patients were included in the study if they met the following criteria: 1) They were at least 20 years of age; 2) Spirometry was performed preoperatively; and 3) They were scheduled to undergo elective cardiac surgery with $\mathrm{CPB}$ and cardioplegic arrest. Patients with concomitant aortic surgery were excluded from participation. The primary endpoints of our study were mechanical ventilation duration, length of postoperative hospital stay, and the 30-day mortality rate.

Patients were classified into 4 ventilation disorder groups, including the normal group ( $\% \mathrm{VC} \geq 80$, FEV $1.0 \% \geq 70$ : $\mathrm{N}$ ), obstructive group (\%VC $\geq 80, \mathrm{FEV} 1.0 \%<70$ : O), restrictive group ( $\% \mathrm{VC}<80, \mathrm{FEV} 1.0 \% \geq 70$ : R), and combined group (\%VC $<80$, FEV1.0\% $<70$ : C), based on the results of the preoperative PFTs. Additionally, we also compared the relationship between \% FEV1.0 and mechanical ventilation duration, length of postoperative hospitalization, and the 30-day mortality rate.
Perioperative management and the operative technique: Following preoperative PFT evaluations, patients outside the normal group were introduced to respiratory interventions, including a medical examination performed by a pulmonologist, cessation of smoking, inhalation of a bronchodilator or steroid, and lung physiotherapy. After hospitalization, all patients were given perioperative breathing training, such as intermittent positive pressure breathing and postoperative rehabilitation by nurses or physical therapists.

Anesthesia, including midazolam $(0.1 \mathrm{mg} / \mathrm{kg})$, rocuronium bromide $(1 \mathrm{mg} / \mathrm{kg})$, and fentanyl $(2 \mu \mathrm{g} / \mathrm{kg})$, intravenously was induced in all patients, according to a standard protocol and maintained by administering a combination of $1.0-2.0 \%$ sevoflurane inhalation (or continuous intravenous administration of propofol $(6-7 \mu \mathrm{g} / \mathrm{kg} / \mathrm{min}$ during $\mathrm{CPB})$, continuous intravenous infusion of remifentanil hydrochloride $(0.1-0.5$ $\mu \mathrm{g} / \mathrm{kg} / \mathrm{min}$ ), and intermittent intravenous administration of rocuronium bromide $(0.2-0.5 \mathrm{mg} / \mathrm{kg})$. Radial and central venous catheters also were introduced. After tracheal intubation, the lungs were ventilated with intermittent positive pressure ventilation. Tidal volume, respiratory frequency, and the fraction of inspired oxygen ( $\mathrm{FiO} 2)$ were adjusted to maintain an arterial $\mathrm{PaCO} 2$ of 35 to $40 \mathrm{mmHg}$ and an arterial oxygen saturation of $>95 \%$.

All patients underwent a single routine surgical technique. After a median sternotomy was performed in all patients, CPB 


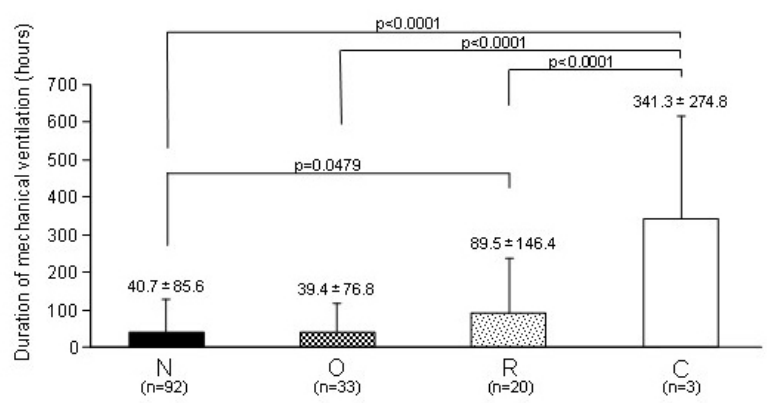

b.

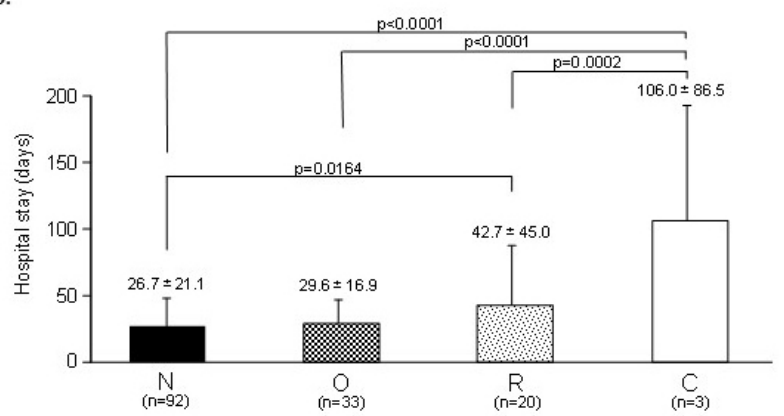

c.

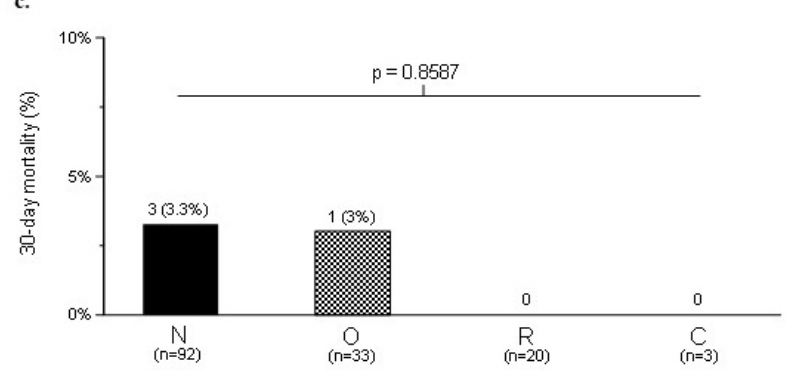

Figure 1. Postoperative outcomes of the study A) The duration of mechanical ventilation expressed in hours; B) Postoperative hospital stay expressed in days; C) Thirty-day mortality expressed as a percentage of incidence. (Abbreviations: $\mathrm{N}$, normal; $\mathrm{O}$, obstructive; $\mathrm{R}$, restrictive; C, combined)

was established and conducted at normothermia $\left(35-36^{\circ} \mathrm{C}\right)$ by ascending the aortic and two stage right atrial or bicaval cannulation, if appropriate. The extracorporeal circuit consisted of a centrifuge pump, capillary membrane oxygenator, hard-shell cardiotomy reservoir, and an arterial filter (MERA Excellung HP, Senko Medical Instrument Co, Ltd., Tokyo, Japan). The circuit was primed with an electrolyte solution (700 mL), mannitol $(200 \mathrm{~mL})$, albumin $(100 \mathrm{~mL})$, sodium bicarbonate (40 mEq), methylprednisolone (1 g), and cefazolin sodium ( $4 \mathrm{~g})$. Heparin was initiated at a dose of $2 \mathrm{mg} /$ $\mathrm{kg}$ and adjusted to achieve a target activated clotting time of $\geq 400$ seconds.

After aortic clamping, $4^{\circ} \mathrm{C}$ cold crystalloid cardioplegia (St. Thomas's Hospital No.2 solution; Miotector®; Mochida Pharmaceutical Co., Tokyo, Japan) was administered bi-directionally at an initial dose of $30 \mathrm{ml} / \mathrm{kg}$ in addition to topical cooling. Next, bidirectional administration of Miotector ${ }^{\circledR}$, which was mixed with blood at a ratio of
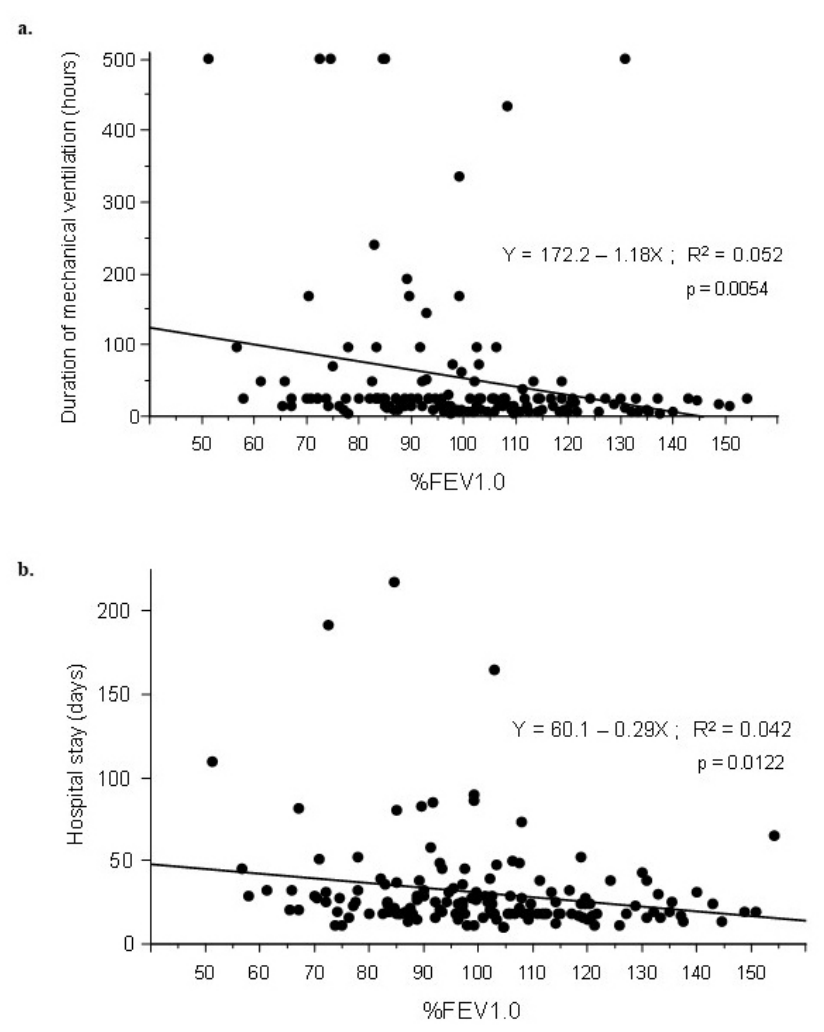

Figure 2. A) The correlation between \%FEV1.0 and the duration of mechanical ventilation; B) The correlation between \%FEV1.0 and the length of hospital stay. (Abbreviation: \%FEV1.0, percent predicted forced expiratory volume in the first second)

$1: 1$, was administered at $10 \mathrm{ml} / \mathrm{kg}$ over 30 -minute intervals. Before aortic de-clamping, terminal warm blood cardioplegia (Hot-shot) was used. Finally, mechanical ventilation was stopped after cardioplegic arrest, and the continuous positive airway pressure (3-4 cm H2O) was maintained during $\mathrm{CPB}$.

Statistical analysis: Categorical parameters are presented as frequencies and percentages unless otherwise described, and continuous variables are presented as the mean \pm standard deviation. Groups were compared using the Pearson chi-square test for categorical variables, and a one-way analysis of variance (or Kruskal-Wallis test for non-parametric data) and post-hoc analyses by Bonferroni-Dunn were used to compare differences. A Pearson product-moment correlation coefficient was computed to assess the relationship between $\%$ FEV1.0 and the mechanical ventilation duration, length of hospital stay, and the 30-day mortality rates. All statistical tests were 2 -tailed, and $P<.05$ was considered statistically significant. All statistical analyses were performed using JMP, version 10.0 (SAS Inc., Cary, NC, USA).

\section{RESULTS}

Patients' characteristics are listed in Table 1. The average patient age was 68 years (range $31-84$ years), and the 
male-female ratio was $87 / 61$. This study consisted of 87 cases of single-valve surgery, 56 cases of multiple valve surgery, 2 cases of intra-cardiac repair for adult congenital heart disease, and 3 cases of cardiac tumor extirpation. There were 23 cases in which concomitant coronary artery bypass surgery was performed.

The perioperative characteristics and features of the patients of the study are shown in the Table. Results from the PFTs revealed that pulmonary dysfunction occurred in nearly $40 \%$ of patients, who underwent elective cardiac surgery. Among these patients, the number of patients assigned to the combined disorder group was smaller than the number of patients assigned to the normal, obstructive, and restrictive groups. Additionally, patients in the combined disorder group were older and had a smaller physique than patients assigned to the other 3 groups, and they were diagnosed with impaired cardiac function before surgery. The mechanical ventilation time in the combined group significantly was longer than in the normal, obstructive, and restrictive groups $(P<.0001$, $P<.0001$, and $P<.0001$, respectively) (Figure 1a). Patients in the combined group also had a significantly longer postoperative hospital stay than patients in the normal, obstructive, and restrictive groups $(P<.0001, P<.0001$, and $P=.0002$, respectively) (Figure 1b). Patients in the restrictive group also had a significantly longer postoperative ventilation or hospitalization than those in the normal group $(P=.0479$, $P=.0164$, respectively) (Figure 1a, b). However, there were no significant differences in 30-day mortality rates between the 4 groups (Figure 1c).

Further, there was a significant negative correlation between \%FEV1.0 and mechanical ventilation $\left(\mathrm{R}^{2}=0.052\right.$, $P=.0054)$ (Figure 2a) and postoperative hospitalization $\left(\mathrm{R}^{2}=0.042, P=.0122\right)$ (Figure $2 \mathrm{~b}$ ), but not with the 30 -day mortality rate.

\section{DISCUSSION}

In clinical settings, preoperative pulmonary function of cardiac patients is commonly screened by spirometry. However, Magee et al. reported that $42 \%$ of COPD patients classified by preoperative PFT improved by $\geq 1$ COPD severity category after aortic valve replacement, including a conventional surgical procedure or transcatheter approach, for severe aortic stenosis [Magee 2013]. Additionally, mortality rates were not significantly different between patients with and without COPD. They also indicated that preoperative measurements by PFT and consequent classification into COPD severity groups overestimated chronic lung disease. Similarly, Ivanov et al suggested that routine PFTs may have limited clinical utility in patients undergoing cardiothoracic surgery when the STS score is readily available because risk stratification was not improved when individual PFT parameters or different COPD classifications were added to the STS scores [Ivanov 2016]. Conversely, Adabag et al demonstrated that PFTs reclassified the COPD status of a significant number or patients after they correlated the clinical history of COPD with the results of PFTs and examined the relationship between preoperative respiratory function and surgical outcomes in a large cohort of patients who underwent cardiac surgery [Adabag 2010].

Additionally, the risk of death was 3.2-fold higher (95\% confidence interval $[\mathrm{CI}], 1.6-6.2, P=.001)$ in patients with moderate or severe airway obstruction (i.e., FEV1.0\% $<0.7$ and $\%$ FEV $1.0<80$ ) than that in patients with no airway obstruction [Adabag 2010]. Patients with moderate to severe airway obstruction also had longer lengths of stay in the ICU and the hospital than patients with no airway obstruction. Further, in a previous high-volume study conducted by Saleh et al., 11217 patients were divided into 3 groups, according to the severity of COPD, which were defined by spirometry and categorized using FEV1.0\% and \%FEV1.0. Their findings revealed that patients with increased COPD severity experienced a significantly higher incidence of postoperative complications, including inotropic support, acute renal failure, and early mortality than did patients with less severe COPD [Saleh 2012]. Results from these studies suggest that it may be important to classify the severity of COPD using \% FEV because FVC decreases as COPD progresses and FEV1.0\% does not accurately reflect the status of COPD. Consistently, this study showed that there was a significant negative correlation between \%FEV1.0 and the length of postoperative hospital stay. Recently, Henn et al demonstrated that moderate or severe respiratory impairment that was predominantly diagnosed using preoperative PFTs was an independent predictor of mortality after surgical aortic valve replacement or transcatheter aortic valve replacement that did not require CPB during surgery [Henn 2016].

Saleh et al reported that a reduced FEV1.0 (odds ratio, 0.99 ; $95 \%$ CI, $0.98-0.99$ ) occurred in $1.96 \%$ of coronary artery bypass grafting patients and that it was a preoperative predictor of prolonged mechanical ventilation [Saleh 2012]. Furthermore, prolonged mechanical ventilation after coronary artery bypass grafting was associated with a reduction in early and mid-term survival. Findings from a study conducted by Zanini et al showed that there was an association between maximal expiratory pressure, which was measured preoperatively as respiratory muscle function, and the duration of invasive mechanical ventilation in patients undergoing coronary artery bypass grafting, valve surgery, and combined procedures [Zanini 2016]. Their study demonstrated that each $1-\mathrm{cm} \mathrm{H} 2 \mathrm{O}$ increase in maximal expiratory pressure reduced the duration of invasive mechanical ventilation by approximately 7 minutes. In this study, prolonged mechanical ventilation occurred in $22(14.9 \%)$ patients, and patients in the combined disorder group had a significantly longer mechanical ventilation duration than patients in the normal, obstructive, or restrictive groups.

Frailty is a deteriorated and complex syndrome that represents a transitional state, during the progression of functional decline [Lang 2009]. Vaz Fragoso et al revealed that there was a strong association between frailty and respiratory dysfunction that was evaluated by spirometry in the elderly [Vaz 2012]. Further, they reported that the coexistence of both factors may increase the risk of death [Vaz 2012]. Hulzebos et al suggested that preoperative inspiratory muscle training reduced 
the incidence of postoperative pulmonary complications and the duration of postoperative hospitalization [Hulzebos 2006]. Further, according to a meta-analysis conducted by Snowdon et al, preoperative interventions, including exercise training, inspiratory muscle training, education, relaxation, counselling, and multiple interventions significantly reduced the time to extubation, relative risk of developing postoperative pulmonary complications (especially in the elderly), and length of ICU and hospital stay [Snowdon 2014].

This study examined the relationship between preoperative pulmonary function classified by spirometry and surgical outcomes after cardiac surgery. The combined ventilation disorder, including both obstructive and restrictive lung disease was significantly associated with prolonged mechanical ventilation and postoperative hospital stay; however, there were no significant differences in the 30-day mortality rates between the 4 ventilation disorder groups. As the number of elderly patients undergoing cardiac surgery increases, opportunities to consider operative procedures for patients with impaired pulmonary function and frailty also increase. Overall, risk stratification by PFTs and the introduction of appropriate perioperative interventions, including education, breathing exercises, and respiratory rehabilitation may be used to accurately identify the preoperative conditions of patients and reduce postoperative respiratory complications or mortality.

There were a few limitations to the current study. First, the retrospective nature and non-randomized observational design limited the current study. Second, this was a single-center study that involved a small number of patients. However, further investigation using the National Clinical Database, which covers all cardiac surgery in Japan, would resolve this. Finally, in this study, we did not include patients undergoing aortic surgery or emergent surgery, which is more invasive and involves a higher risk.

\section{CONCLUSION}

Preoperative PFT revealed that pulmonary dysfunction occurred in nearly $40 \%-50 \%$ of patients undergoing elective cardiac surgery with CPB. Specifically, the mechanical ventilation duration and length of postoperative hospital stay were significantly longer in the combined lung disease group than those in the normal, obstructive, and restrictive groups. The addition of \% FEV to the COPD severity might appropriately predict the postoperative morbidity or clinical course.

\section{REFERENCES}

Ad N, Henry L, Halpin L, et al. 2010. The use of spirometry testing prior to cardiac surgery may impact the Society of Thoracic Surgeons risk prediction score: a prospective study in a cohort of patients at high risk for chronic lung disease. J Thorac Cardiovasc Surg 139:686-91.

Adabag AS, Wassif HS, Rice K, et al. 2010. Preoperative pulmonary function and mortality after cardiac surgery. Am Heart J 159:691-7.

Celli BR, MacNee W, ATS/ERS Task Force. 2004. Standards for the diagnosis and treatment of patients with COPD: a summary of the ATS/ ERS position paper. Eur Respir J 23:932-46.

Cohen AJ, Katz MG, Frenkel G, et al. 2000. Morbid results of prolonged intubation after coronary artery bypass surgery. Chest 118:1724-31.

Filsoufi F, Rahmanian PB, Castillo JG, Chikwe J, Adams DH. 2008. Predictors and early and late outcomes of respiratory failure in contemporary cardiac surgery. Chest 133:713-21.

Henn MC, Zajarias A, Lindman BR, et al. 2016. Preoperative pulmonary function tests predict mortality after surgical or transcatheter aortic valve replacement. J Thorac Cardiovasc Surg 151:578-85.

Hulzebos EH, Helders PJ, Favié NJ, et al. 2006. Preoperative intensive inspiratory muscle training to prevent postoperative pulmonary complications in high-risk patients undergoing CABG surgery: a randomized clinical trial. JAMA 296:1851-7.

Ivanov A, Yossef J, Tailon J, et al. 2016. Do pulmonary function tests improve risk stratification before cardiothoracic surgery? J Thorac Cardiovasc Surg 151:1183-9.

Lang PO, Michel JP, Zekry D. 2009. Frailty syndrome: a transitional state in a dynamic process. Gerontology 55:539-49.

Magee MJ, Herbert MA, Roper KL, et al. 2013. Pulmonary function tests overestimate chronic pulmonary disease in patients with severe aortic stenosis. Ann Thorac Surg 96:1329-35.

Mannino DM, Buist AS, Petty TL, Enright PL, Redd SC. 2003. Lung function and mortality in the United States: data from the First National Health and Nutrition Examination Survey follow up study. Thorax 58:388-93.

Miyata H, Tomotaki A, Motomura N, Takamoto S. 2015. Operative mortality and complication risk model for all major cardiovascular operations in Japan. Ann Thorac Surg 99:130-9.

Roques F, Nashef SA, Michel P, et al. 1999. Risk factors and outcome in European cardiac surgery: analysis of the EuroSCORE multinational database of 19030 patients. Eur J Cardiothorac Surg 15:816-22.

Saleh HZ, Mohan K, Shaw M, et al. 2012. Impact of chronic obstructive pulmonary disease severity on surgical outcomes in patients undergoing non-emergent coronary artery bypass grafting. Eur J Cardiothorac Surg 42:108-13.

Saleh HZ, Shaw M, Al-Rawi O, et al. 2012. Outcomes and predictors of prolonged ventilation in patients undergoing elective coronary surgery. Interact Cardiovasc Thorac Surg 15:51-6.

Samuels LE, Kaufman MS, Morris RJ, Promisloff R, Brockman SK. 1998. Coronary artery bypass grafting in patients with COPD. Chest113:878-82.

Shroyer AL, Coombs LP, Peterson ED, et al. 2003. The Society of Thoracic Surgeons: 30 -day operative mortality and morbidity risk models. Ann Thorac Surg 75:1856-64.

Snowdon D, Haines TP, Skinner EH. 2014. Preoperative intervention reduces postoperative pulmonary complications but not length of stay in cardiac surgical patients: a systematic review. J Physiother 60:66-77.

Vaz Fragoso CA, Enright PL, McAvay G, Van Ness PH, Gill TM. 2012. Frailty and respiratory impairment in older persons. Am J Med 125:79-86.

Zanini M, Nery RM, Buhler RP, de Lima JB, Stein R. 2016. Preoperative maximal expiratory pressure is associated with duration of invasive mechanical ventilation after cardiac surgery: An observational study. Heart Lung 45:244-8. 